Article

\title{
Absorbability, Mechanism and Structure-Property Relationship of Three Phenolic Acids from the Flowers of Trollius chinensis
}

\author{
Xiu-Wen Wu, Ru-Feng Wang *, Li-Jia Liu, Li-Na Guo and Can Zhao \\ School of Chinese Materia Medica, Beijing University of Chinese Medicine, Beijing 100102, China; \\ E-Mails: wuxiuwen0725@126.com (X.-W.W.); 1ily199109231@163.com (L.-J.L.); \\ gngn2010@163.com (L.-N.G.); 15201391380@163.com (C.Z.) \\ * Author to whom correspondence should be addressed; E-Mail: wangrufeng@tsinghua.org.cn; \\ Tel./Fax: +86-10-8473-8646.
}

External Editor: Derek J. McPhee

Received: 18 July 2014; in revised form: 1 October 2014 / Accepted: 27 October 2014/

Published: 5 November 2014

\begin{abstract}
The absorption properties, mechanism of action, and structure-property relationship of three phenolic acids isolated from the flowers of Trollius chinensis Bunge, namely, proglobeflowery acid (PA), globeflowery acid (GA) and trolloside (TS), were investigated using the human Caco-2 cell monolayer model. The results showed that these three phenolic acids were transported across the Caco-2 cell monolayer in a time and concentration dependent manner at the $\mathrm{P}_{\text {app }}$ level of $10^{-5} \mathrm{~cm} / \mathrm{s}$, and their extent of absorption correlated with their polarity and molecular weight. In conclusion, all three of these compounds were easily absorbed through passive diffusion, which implied their high bioavailability and significant contribution to the effectiveness of $T$. chinensis.
\end{abstract}

Keywords: Trollius chinensis; Ranunculaceae; phenolic acids; absorbability; Caco-2 cell monolayer model

\section{Introduction}

Proglobeflowery acid (systematic name: 4-hydroxy-3-methoxy-5-(3-methylbut-2-en-1-yl)benzoic acid) (PA), globeflowery acid (systematic name: 8-methoxy-2,2-dimethylchroman-6-carboxylic acid) (GA) and trolloside (systematic name: 3-methoxy-5-(3-methylbut-2-en-1-yl)-4-(((2S,3R,4S,5S,6R)- 
3,4,5-trihydroxy-6-(hydroxymethyl)tetrahydro-2H-pyran-2-yl)oxy)benzoic acid) (TS) are three important phenolic acids isolated from the flowers of Trollius chinensis Bunge which mainly contain phenolic acids, flavonoids and alkaloids [1-3]. Structurally, these three phenolic acids are $p$-hydroxy acid derivatives. PA and GA are isomers, the former being intramolecularly cyclized into the latter under acidic condition, while TS is the glycosylated form of PA (Figure 1). Pharmacological studies have demonstrated that phenolic acids, including these three compounds, exhibit various biological activities, which are closely related to the effect of these flowers in the treatment of respiratory infections, pharyngitis, tonsillitis and bronchitis. For example, PA showed inhibitory activity against Pseudomonas aeruginosa and Staphylococcus aureus with MIC values of 16 and $200 \mathrm{mg} / \mathrm{L}$, respectively [4,5], and also had weak antiviral activity against Para 3 with an $\mathrm{IC}_{50}$ value of $184.2 \mu \mathrm{g} / \mathrm{mL}$ [6]. GA exhibited significant antiviral activity against influenza A with an IC50 value of $42.1 \mu \mathrm{g} / \mathrm{mL}$, and TS displayed moderate inhibitory effect against Streptococcus pneumonia with a MIC value of $128 \mathrm{mg} / \mathrm{L}$ [7]. Thus, these three phenolic acids can be considered as the bioactive components of the flowers of $T$. chinensis. However, to what extent they are effective in the human body depends on their bioavailability and metabolism in vivo. As is well known, absorbability is a crucial factor which influences the bioavailability of drugs, especially of those being administrated orally. For this reason, the present study investigated the absorption property and mechanism, as well as structure-absorbability relationship of these three phenolic acids using the well-recognized human Caco-2 monolayer model, so as to predict their bioavailability and understand their contribution to the effectiveness of these flowers.

Figure 1. Chemical structures of PA, GA and TS.



\section{Results and Discussion}

\subsection{Results}

\subsubsection{Validation of HPLC Analytical Method}

The HPLC chromatograms of the analytes (Figure 2) showed that all chromatographic peaks of interest were well resolved at baseline under the optimal conditions. All these analytes were quantified by linear regression analysis, and their contents were all within the linear limits. The RSD values for intra-day and inter-day precision and stability tests were all well below $2.3 \%$, indicating that the analytical method employed was validated and the samples were stable under the test condition. 
Figure 2. HPLC chromatograms of PA, GA and TS.
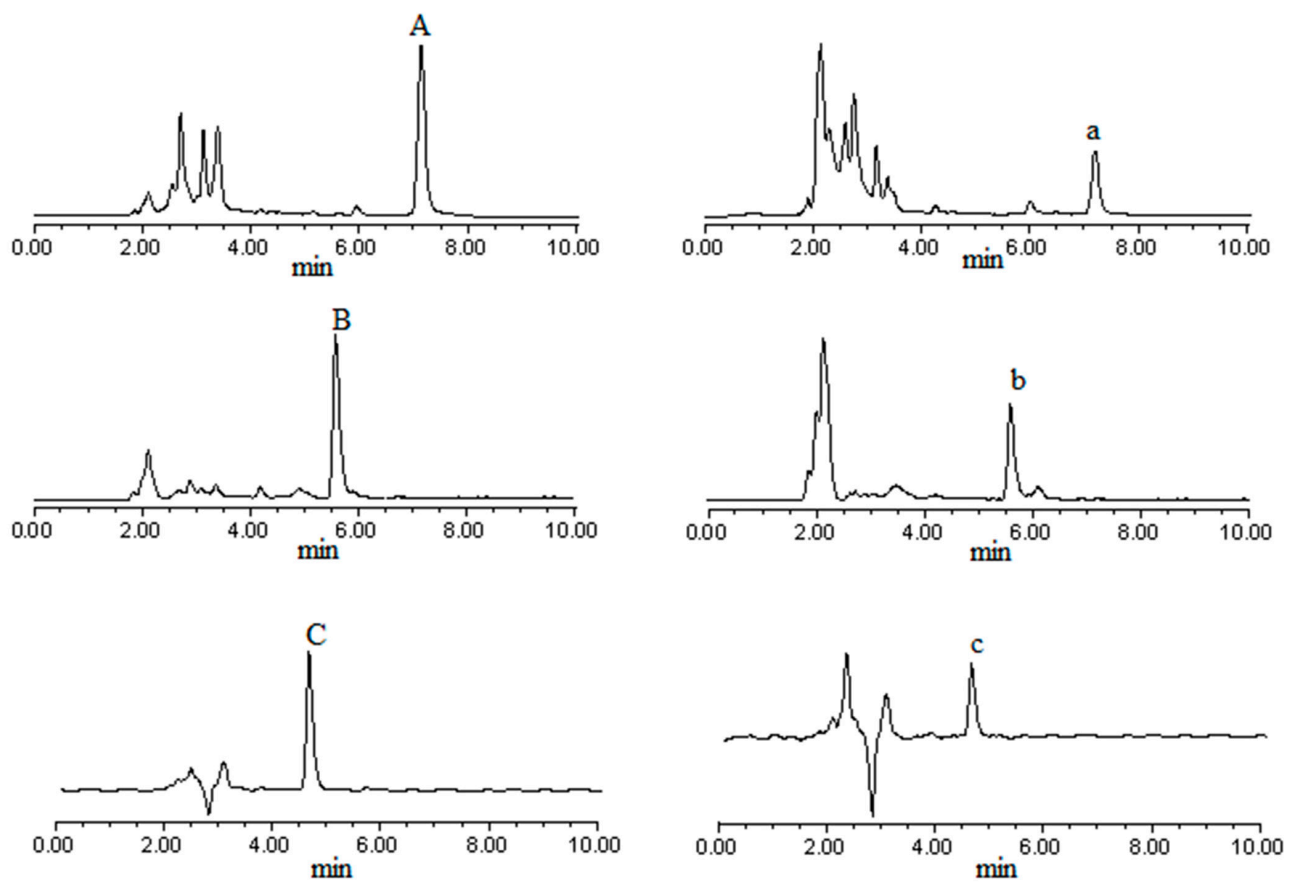

Notes: A, B, C were chromatograms of reference solutions of PA, GA and TS; $a, b$ and $\mathrm{c}$ were chromatograms of sample solutions of PA, GA and TS across the monolayer.

\subsubsection{Validation of Caco-2 Monolayer Model}

The TEER values of the monolayers developed in this study increased steadily over time and reached over $600 \Omega \cdot \mathrm{cm}^{2}$ on day 21 after seeding. The Papp values of reference controls, propranolol and atenolol, tested with the monolayers in this study were $(1.24 \pm 0.10) \times 10^{-5} \mathrm{~cm} / \mathrm{s}$ and $(6.92 \pm 0.51) \times 10^{-7} \mathrm{~cm} / \mathrm{s}$ respectively, which were in good agreement with the acceptable values reported in the literature [8]. Thus, the Caco-2 cell monolayer model was validated for assessment of the intestinal absorption potential of the compounds of interest.

\subsubsection{Non-Toxic Dose Range of Test Compounds}

The survival rates of Caco- 2 cells treated with three phenolic acids at different concentrations in MTT assay (Table 1) was all above $95 \%$ and no cytotoxicity was observed, whereas those of the Caco- 2 cells treated with paclitaxel (the positive control) were below $70 \%$ and exhibited dose dependence. These results showed that the Caco- 2 cells were not influenced by the test compounds at the concentrations up to $100 \mu \mathrm{mol} / \mathrm{L}$. Therefore, 25,50 and $100 \mu \mathrm{mol} / \mathrm{L}$ were chosen as the test concentrations.

Table 1. Survival rates of Caco-2 cells treated with test compounds $(n=4)$.

\begin{tabular}{cccc}
\hline \multirow{2}{*}{ Compounds } & \multicolumn{3}{c}{ Survival Rate: $\%$} \\
\cline { 2 - 4 } & $\mathbf{2 5} \boldsymbol{\mu \mathbf { m o l } / \mathbf { L }}$ & $\mathbf{5 0} \boldsymbol{\mu \mathbf { m o l }} / \mathbf{L}$ & $\mathbf{1 0 0} \boldsymbol{\mu m o l} / \mathbf{L}$ \\
\hline PA & $118.8 \pm 11.6$ & $110.5 \pm 3.8$ & $124.5 \pm 2.2$ \\
GA & $107.1 \pm 5.7$ & $118.9 \pm 13.7$ & $123.6 \pm 6.2$ \\
TS & $98.9 \pm 4.3$ & $107.3 \pm 7.0$ & $118.2 \pm 8.1$ \\
Paclitaxel & $67.3 \pm 8.2$ & $45.5 \pm 6.7$ & $37.4 \pm 3.5$ \\
\hline
\end{tabular}




\subsubsection{Transport of Test Compounds}

Under the conditions of this experiment, the human intestinal permeability of PA, GA and TS was evaluated using Caco-2 cell monolayer model. The transport was monitored for a period of $90 \mathrm{~min}$. The bidirectional, i.e., both from apical (AP) to basolateral (BL) side and from BL to AP side apparent permeability coefficients ( $\mathrm{P}_{\text {app}}$ ) values of PA, GA and TS (Table 2 ) were all at the level of $10^{-5} \mathrm{~cm} / \mathrm{s}$.



\begin{tabular}{|c|c|c|c|c|}
\hline \multicolumn{2}{|c|}{ Concentration $(\mu \mathrm{mol} / \mathrm{L})$} & $\mathbf{P}_{\text {app AP } \rightarrow \mathrm{BL}}$ & $\mathbf{P}_{\text {app BL } \rightarrow \text { AP }}$ & $\mathbf{P}_{\text {ratio }}$ \\
\hline \multirow{4}{*}{ PA } & 25 & $3.17 \pm 0.04$ & $2.54 \pm 0.22$ & 1.25 \\
\hline & 50 & $2.80 \pm 0.13$ & $2.56 \pm 0.19$ & 1.1 \\
\hline & 100 & $2.96 \pm 0.11$ & $2.59 \pm 0.14$ & 1.14 \\
\hline & average & $2.98 \pm 0.13$ & $2.56 \pm 0.18$ & 1.16 \\
\hline \multirow{4}{*}{ GA } & 25 & $2.14 \pm 0.20$ & $2.21 \pm 0.08$ & 0.96 \\
\hline & 50 & $1.59 \pm 0.02$ & $2.00 \pm 0.07$ & 0.8 \\
\hline & 100 & $1.70 \pm 0.12$ & $1.94 \pm 0.10$ & 0.88 \\
\hline & average & $1.81 \pm 0.11$ & $2.05 \pm 0.08$ & 0.88 \\
\hline \multirow{4}{*}{ TS } & 25 & $0.86 \pm 0.16$ & $0.86 \pm 0.04$ & 1 \\
\hline & 50 & $1.06 \pm 0.03$ & $1.08 \pm 0.06$ & 0.98 \\
\hline & 100 & $0.77 \pm 0.09$ & $0.80 \pm 0.03$ & 0.96 \\
\hline & average & $0.89 \pm 0.09$ & $0.91 \pm 0.04$ & 0.98 \\
\hline
\end{tabular}

Notes: $\mathrm{P}_{\text {app AP } \rightarrow \mathrm{BL}}$ : transport of test compounds from AP to BL; $\mathrm{P}_{\text {app BL } \rightarrow \mathrm{AP}}$ : transport of test compounds from BL to AP; $\mathrm{P}_{\text {ratio }}\left(\mathrm{P}_{\text {app AP } \rightarrow \mathrm{BL}} / \mathrm{P}_{\text {app BL } \rightarrow \mathrm{AP}}\right)$ : the ratio of $\mathrm{P}_{\text {app } \mathrm{AP} \rightarrow \mathrm{BL}}$ to $\mathrm{P}_{\text {app BL } \rightarrow \mathrm{AP}}$. All data was expressed as the mean $\pm \mathrm{SD}(\mathrm{n}=3)$. The incubation time was up to $90 \mathrm{~min}$.

\subsubsection{Time Course}

The approximate kinetic curves of these three compounds over time (30-180 min, Figure 3) showed that their percentage of transport increased with the incubation time in an approximate linear manner.

Figure 3. Transport of three phenolic acids across Caco-2 cell monolayer as the function of time at $50 \mu \mathrm{mol} / \mathrm{L}$ (a) AP to BL side; (b) BL to AP side (means $\pm \mathrm{SD}, \mathrm{n}=3$ ).
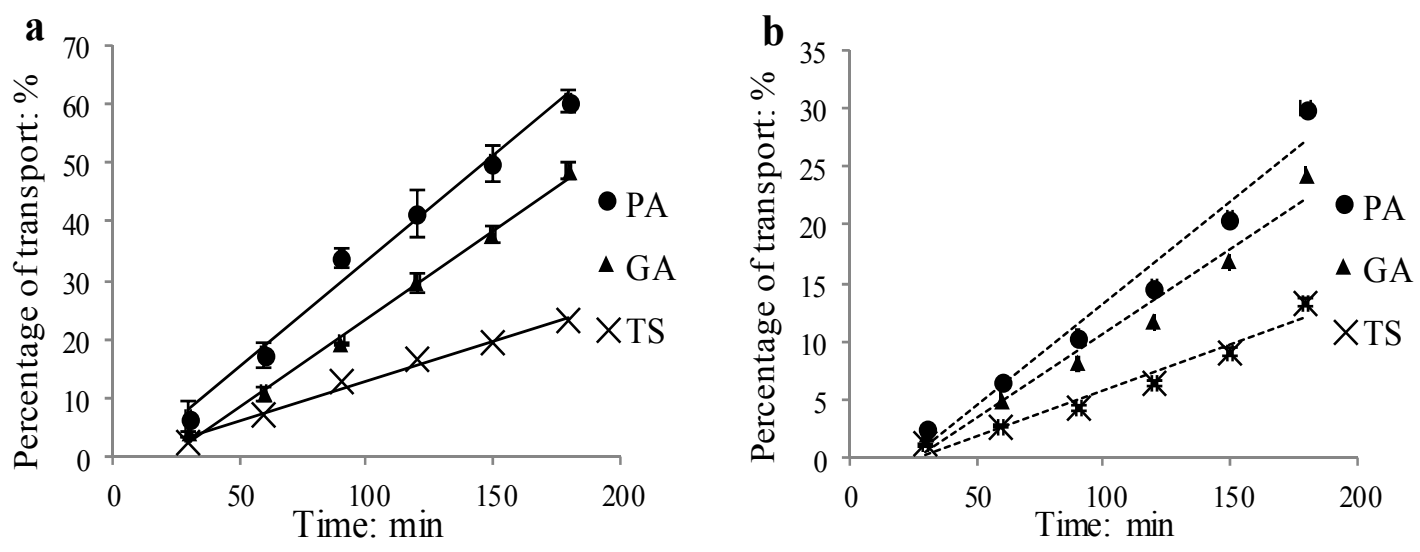


\subsubsection{Concentration Dependence}

The kinetic curves of these three compounds over concentration (25-100 $\mu \mathrm{mol} / \mathrm{L}$, Figure 4) showed that their bidirectional transport rate increased with concentration in an approximately linear manner.

Figure 4. Transport of three phenolic acids across Caco-2 monolayer as the function of concentration at 90 min (a) AP to BL side; (b) BL to AP side (means $\pm \mathrm{SD}, \mathrm{n}=3$ ).
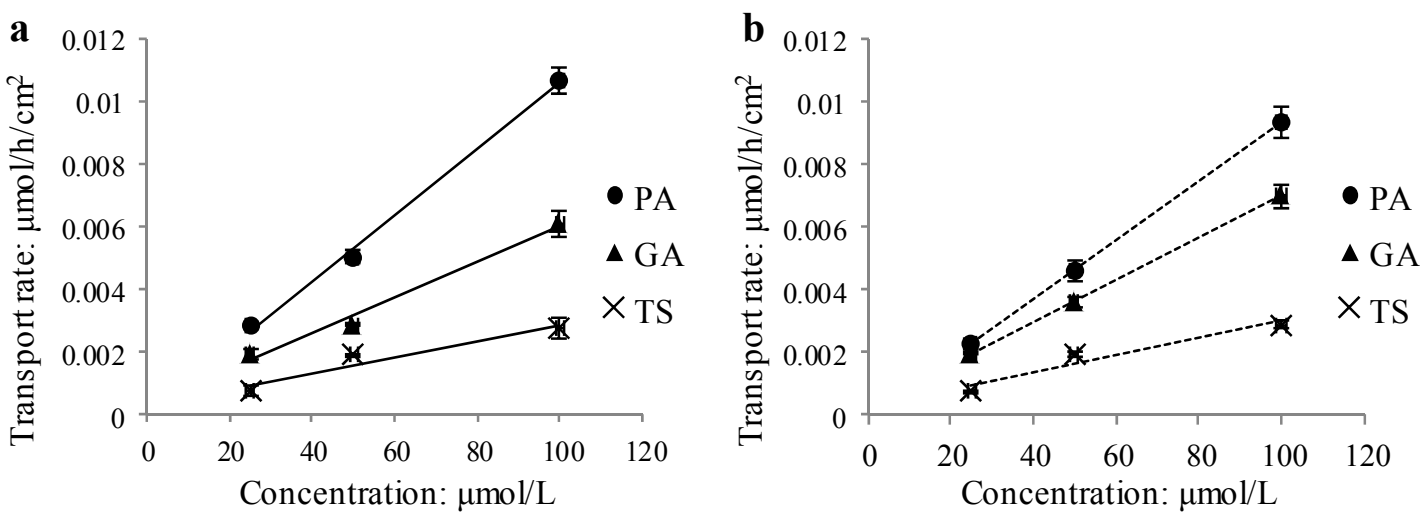

\subsubsection{Molecular Properties}

The molecular properties of PA, GA and TS calculated by software are listed in the Table 3 .

Table 3. Molecular properties of PA, GA and TS.

\begin{tabular}{cccccccc}
\hline Compound & Molecular Mass & LogP & TPSA & $\mathbf{H}_{\mathbf{d}}$ & $\mathbf{H}_{\mathbf{r}}$ & $\mathbf{R}$ & $\mathbf{V}$ \\
\hline PA & 236.26 & 2.67 & 66.761 & 2 & 4 & 4 & 0 \\
GA & 236.26 & 2.27 & 55.767 & 1 & 4 & 2 & 0 \\
TS & 398.4 & 0.83 & 145.913 & 5 & 9 & 7 & 0 \\
\hline
\end{tabular}

Notes: $\mathrm{H}_{\mathrm{d}}$ : the number of hydrogen bond donor; $\mathrm{H}_{\mathrm{r}}$ : the number of hydrogen bond receptor; $\mathrm{R}$ : the number of rotatable bond; V: the number of violation of the "five rules".

\subsection{Discussion}

Under the conditions of the experiments, the human intestinal permeability of PA, GA and TS was evaluated using the Caco-2 cell monolayer model. The Papp values of PA, GA and TS were at the same magnitude $\left(10^{-5} \mathrm{~cm} / \mathrm{s}\right)$ as that of propranolol, which is often used as a reference compound of high permeability [8-10]. This implied that these compounds were easily absorbed by human body through intestinal mucosa. It has been well known that "five rules" $\left(\mathrm{H}_{\mathrm{d}}<5 ; \mathrm{H}_{\mathrm{r}}<10 ; \operatorname{LogP}<5\right.$; Molecular mass $<500$; passive transport) and "Veber rules" (TPSA $<140 \AA^{2} ; \mathrm{R}<10$ ) [11] are useful to evaluate the absorption of compounds. According to these rules, none of these three phenolic acids violate the "five rules". Unlike PA and GA, however, TS (TPSA $=146 \AA^{2}$ ) (Table 3) violates the "Veber rules". Thus, all of these three compounds were easily absorbed, but TS was the relatively poorest absorbed one among them. As a matter of fact, the absorption extent of these three compounds correlated with their molecular polarity and molecular weight. In general, the larger the molecular polarity and molecular weight of the compound, the more difficult it is absorbed. Undoubtedly, TS is 
the highest in either polarity or molecular weight. Correspondingly, the bidirectional $\mathrm{P}_{\text {app }}$ values of this compound were the lowest among those of the three compounds. As for PA and GA, they are isomers with the same molecular weight. In this case, molecular polarity is the crucial factor to determine the absorption order of difficulty. PA is less polar than GA, which is evidenced by the RP-HPLC chromatogram of these compounds (Figure 2) and the LogP values (Table 3). Accordingly, the larger

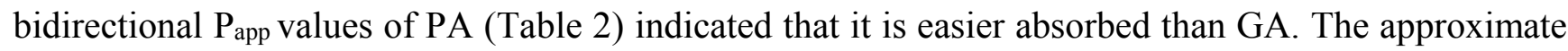
linear increase of bidirectional transport or transport rate of these three compounds over time and concentration (Figures 3 and 4) demonstrated that they were across the monolayer mainly through passive transcellular diffusion. Furthermore, it was confirmed by the fact that the $\mathrm{P}_{\mathrm{app}} \mathrm{AP} \rightarrow \mathrm{BL}$ to

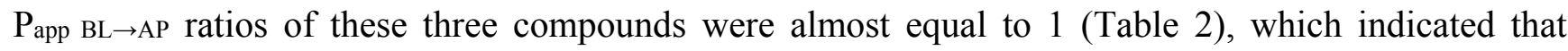
neither efflux nor active transport was involved in their absorption.

On the basis of the literature [8], the test phenolic acids would be absorbed over $70 \%$ after oral application in vivo. In our previous study, the most concentrated phenolic acid in the flowers of T. chinensis, i.e., veratric acid was demonstrated to be well absorbed. All in all, the phenolic acids in the flowers of $T$. chinensis have a good absorbability; thus, these compounds should be attached importance in the evaluation of effective components of these flowers.

\section{Experimental}

\subsection{General}

Dulbecco's Modified Eagle's Medium (DMEM), fetal bovine serum (FBS) and trypsin were supplied by Gibco (Grand Island, CA, USA). Nonessential amino acids (NEAA), penicillin and streptomycin were purchased from Corning Costar (Cambridge, MA, USA). 3-(4,5-dimethylthiazolzyl)-2,5-diphenyl tetrazolium bromide (MTT), ethylene diaminetetraacetic acid (EDTA), dimethyl sulfoxide (DMSO), propranolol and atenolol with purity of minimum 98\% were products of Sigma Chemical Co. (Deisenhofen, Germany). Proglobeflowery acid, globeflowery acid and trolloside were isolated from the flowers of $T$. chinensis by our research group and their purities were determined to be over $98 \%$ by HPLC. Paclitaxel drug product (Batch No. 20140201, Strength $30 \mathrm{mg} / 5 \mathrm{~mL}$ ) was provided by Beijing SL Pharmaceutical Co., Ltd. (Beijing, China). Other chemicals were of analytical grade and solvents used in high-performance liquid chromatography (HPLC) were of HPLC grade. Transwell plates (insert diameter $12 \mathrm{~mm}$, pore size $3.0 \mu \mathrm{m}$, membrane growth area $1.12 \mathrm{~cm}^{2}$ ) were purchased from Corning Costar.

\subsection{Plant Material}

The flowers of $T$. chinensis were purchased from the Anguo drug market of Hebei Province and identified by Ru-Feng Wang. A voucher specimen (No. 20120526) has been deposited at the Herbarium of School of Chinese Materia Medica, Beijing University of Chinese Medicine.

\subsection{Extraction and Isolation of GA, PA and TS}

About $2 \mathrm{~kg}$ of the dried flowers of $T$. chinensis was extracted with $36 \mathrm{~L}$ of $95 \%$ ethanol under reflux for $2 \mathrm{~h}$. The extract $(0.6 \mathrm{~kg})$ was concentrated in vacuo, and then was suspended in $1.2 \mathrm{~L}$ of water. The 
suspension was partitioned successively with each $1.2 \mathrm{~L}$ of petroleum ether, ethyl acetate and $n$-butanol. The resultant EtOAc-soluble part (50 g) was separated by polyamide (30 60 mesh) column chromatography eluted with $\mathrm{H}_{2} \mathrm{O}, 20 \% \mathrm{MeOH}, 50 \% \mathrm{MeOH}, 70 \% \mathrm{MeOH}$ and $95 \% \mathrm{MeOH}$, respectively, to afford five major fractions (Fr.A-Fr.E). Fr.C (2.1 g) was subjected to silica gel chromatography eluted with gradient $\mathrm{CHCl}_{3}-\mathrm{MeOH}$ from 30:1 to 1:1 to give five fractions (Fr.C1 Fr.C5). Fr.C2 (310 mg) was further isolated with silica gel column chromatography eluted with $\mathrm{CHCl}_{3}-\mathrm{MeOH}$ from 30:1 to 10:1 to afford PA (14 mg) and GA $(15 \mathrm{mg})$. Fr.C3 (0.93 g) was isolated with silica gel column chromatography using $\mathrm{CHCl}_{3}-\mathrm{MeOH}$ from 30:1 to 2:1 as eluent to obtain TS (82 mg).

\subsection{Cell Culture}

The human colon adenocarcinoma cell line Caco-2 was purchased from the Cell Resource Center, Peking Union Medical College (CRC/PUMC, Beijing, China), and the Caco-2 cells between passages 32 and 36 were cultured in $25 \mathrm{~cm}^{2}$ flasks in DMEM medium supplemented with $10 \%$ (v/v) FBS, $1 \%$ (v/v) NEAA, $1 \%(\mathrm{v} / \mathrm{v})$ penicillin-streptomycin, in an atmosphere of $5 \% \mathrm{CO}_{2}$ and $95 \%$ air at $37{ }^{\circ} \mathrm{C}$ and constant humidity. The culture medium was replaced every 2-3 days, and the cells were split 1:2 or 1:3 when they reached $80 \%$ confluence.

\subsection{MTT Assay}

Caco-2 cells at logarithmic growth phase were seeded onto 96 -well plates and incubated at $37{ }^{\circ} \mathrm{C}$ in culture hood. The culture medium was removed after cells formed a full layer, and each $100 \mu \mathrm{L}$ of HBSS solution (negative control group) and drug-containing HBSS solution (three phenolic acids at the concentrations of 25,50 and $100 \mu \mathrm{mol} / \mathrm{L}$, respectively) was added to each well. When the plates were cultured for $4 \mathrm{~h}$, HBSS solution was completely transferred out and each $100 \mu \mathrm{L}$ of MTT solution $(0.5 \mathrm{mg} / \mathrm{mL})$ was added to each well. MTT solution was removed carefully after 4 hours of incubation at $37^{\circ} \mathrm{C}$, and each $150 \mu \mathrm{L}$ of DMSO was added to each well, then culture vessels were agitated on an orbital shaker for $10 \mathrm{~min}$ and absorbance was read on a microplate reader at $570 \mathrm{~nm}$.

\subsection{Cell Differentiation}

To facilitate the cells' differentiation and formation of a confluent monolayer, Caco-2 cells were seeded at a density of $1.0 \times 10^{5}$ cells $/ \mathrm{cm}^{2}$ on a 12 -well Transwell insert filter. The inserts were fed with culture medium at 2 days intervals in the first week, and then at daily intervals for the AP side and 2 days intervals for the BL side until they were used for the transport experiment 21 days after seeding. The AP and BL sides contained $0.5 \mathrm{~mL}$ and $1.5 \mathrm{~mL}$ of culture medium, respectively. The integrity and viability of the cell monolayers were evaluated by measuring TEER values in culture medium at $37{ }^{\circ} \mathrm{C}$ using Millicell ${ }^{\circledR}$-ERS system (Millipore Corp., Bedford, MA, USA) and transport experiment using standard compounds, i.e., propranolol and atenolol which were well-known control substances for high and poor transcellular transport markers, respectively. The cell inserts were used after the resistance reached above $600 \Omega \cdot \mathrm{cm}^{2}$. 


\subsection{Transport Experiment}

On day 21, transport study was initiated by careful removal of the culture medium from AP and BL sides. Caco- 2 monolayers were rinsed twice with pre-warmed HBSS and were incubated by pre-warmed HBSS for $30 \mathrm{~min}$ at $37^{\circ} \mathrm{C}$. PA, GA and TS were dissolved in DMSO, and diluted to the concentration of 25,50 and $100 \mu \mathrm{mol} / \mathrm{L}$ with HBSS. The final DMSO concentration was less than $1 \%$. Test compounds were added to the AP side $(0.5 \mathrm{~mL})$ or BL side $(1.5 \mathrm{~mL})$ of the inserts, while the receiving chamber contained the corresponding volume of HBSS. Standard markers (propranolol and atenolol) were just added to the AP side $(0.5 \mathrm{~mL})$. Incubation was performed at $37^{\circ} \mathrm{C}$ for $180 \mathrm{~min}$, with shaking at $50 \mathrm{rpm}$. At 30,60, 90, 120, 150 and $180 \mathrm{~min}, 0.2 \mathrm{~mL}$ of solutions from BL side or AP side were collected, and replaced with an equal volume of HBSS. Samples were frozen immediately and stored below $-20{ }^{\circ} \mathrm{C}$ before analysis. Upon completion of all permeation experiments, TEER values were measured to ensure that cell monolayer integrity and viability had not been adversely affected by experimental conditions.

\subsection{HPLC Analysis}

\subsubsection{Chromatographic Condition}

PA, GA and TS were determined on Waters 1500 system consisting of a 1525 Binary HPLC pump, a $2489 \mathrm{UV} /$ Visible detector, an on-line degasser and a manual injector connecting a $20 \mu \mathrm{L}$ loop. The signals were acquired and processed using Windows XP-based Waters Breeze 2 software. HPLC analysis was performed on a Kromasil $\mathrm{C}_{18}$ ODS column $(250 \mathrm{~mm} \times 4.60 \mathrm{~mm}$ i.d., $5 \mu \mathrm{m}$ particle size) with a guard column. For PA and GA, the mobile phase consisted of acetonitrile and $1 \%$ acetic acid $(50: 50, \mathrm{v} / \mathrm{v})$ at a flow rate of $1.00 \mathrm{~mL} / \mathrm{min}$, UV detector was set at $269 \mathrm{~nm}$ and $251 \mathrm{~nm}$ respectively, and temperature was $35{ }^{\circ} \mathrm{C}$. For TS, the mobile phase consisted of acetonitrile and $1 \%$ acetic acid $(30: 70, \mathrm{v} / \mathrm{v})$ at a flow rate of $1.00 \mathrm{~mL} / \mathrm{min}$, UV detector was set at $251 \mathrm{~nm}$ and temperature was $35{ }^{\circ} \mathrm{C}$. Each $20 \mu \mathrm{L}$ of sample was injected into the HPLC after filtered through $0.45 \mu \mathrm{m}$ Millipore filters. The chromatograms of the three phenolic acids are shown in Figure 2.

\subsubsection{Linearity, Precision and Stability}

The calibration curves were constructed by plotting peak area (Y, mAU*min) vs. amount (X, in $\mu$ mol). The linear equation of three phenolic acids were as follows: for PA, $\mathrm{Y}=1.02 \times 10^{8} \mathrm{X}+484(\mathrm{r}=0.9999)$ with a good linearity over the range from $2.5 \times 10^{-5} \mu \mathrm{mol}$ to $2.0 \times 10^{-4} \mu \mathrm{mol}$; for GA, $\mathrm{Y}=1.57 \times 10^{8} \mathrm{X}+372(\mathrm{r}=0.9994)$ with a good linearity over the range from $1.25 \times 10^{-5} \mu \mathrm{mol}$ to $5.0 \times 10^{-4} \mu \mathrm{mol}$; for TS, Y $=3.13 \times 10^{8} \mathrm{X}-271(\mathrm{r}=0.9996)$ with a good linearity over the range from $1.0 \times 10^{-6} \mu \mathrm{mol}$ to $2.5 \times 10^{-4} \mu \mathrm{mol}$. Quantification was carried out by peak area measurements in comparison with the calibration curves.

Precisions were determined by investigating the reference solutions of PA, GA and TS in sextuplicates during a single day as intra-day precision and duplicating the intra-day experiment on two consecutive days as inter-day precision. The RSD values of intra-day precision for the three analytes were $0.57 \%, 1.20 \%$ and $1.58 \%$, respectively, while those values of inter-day precision for the 
three analytes were $0.62 \%, 1.19 \%$ and $2.17 \%$, respectively. Each one sample solution of PA, GA and TS was kept at room temperature, and then the stability was determined by injecting it into apparatus at $0,1,2,4,6,12$ and $24 \mathrm{~h}$. The RSD values for 24-hour stability of the three analytes were $1.69 \%$, $2.03 \%$ and $2.26 \%$, respectively. It demonstrated that the samples were stable within $24 \mathrm{~h}$.

\subsection{Calculation of Molecular Properties}

The logarithm of octanol-water partition coefficient $(\log P)$ was assessed with ChemOffice 2004 (CambridgeSoft Corporation, Cambridge, MA, USA), and the other properties, such as TPSA, $\mathrm{H}_{\mathrm{d}}$ (the number of hydrogen bond donor), $\mathrm{H}_{\mathrm{r}}$ (the number of hydrogen bond receptor), $\mathrm{R}$ (the number of rotatable bond), and V (the number of violation of the "five rules"), were acquired from online products of Molinspiration Corporation. The results are listed in Table 3.

\subsection{Data Analysis}

The cell survival rate $(\%)$ was calculated using the equation survival rate $=\overline{\mathrm{A}} / \overline{\mathrm{A}_{0}} \times 100$, wherein $\overline{\mathrm{A}}$ was the average absorbance value of treatment group; $\overline{\mathrm{A}_{0}}$ was the average absorbance value of the blank control group. The $\mathrm{P}_{\mathrm{app}}$ was determined in this study by HPLC quantification of the compounds in the receiver chamber after transport across the Caco-2 monolayer. The calculation was described by the equation $\mathrm{P}_{\mathrm{app}}=(\mathrm{dQ} / \mathrm{dt}) \times(1 / \mathrm{A}) \times\left(1 / \mathrm{C}_{0}\right)$, wherein $\mathrm{dQ} / \mathrm{dt}$ was the rate of appearance of the test compound on the receiver compartment $(\mu \mathrm{mol} / \mathrm{s}) ; \mathrm{C}_{0}$ was the initial test compound concentration on the donor compartment $(\mu \mathrm{mol} / \mathrm{mL})$; and A was the surface area of Caco-2 monolayer $\left(\mathrm{cm}^{2}\right)$.

The percentage transported (\%) was calculated using the equation $\%$ Transported $=\mathrm{Q}_{\mathrm{B}} /\left(\mathrm{CS}_{\mathrm{S}} \times \mathrm{V}_{\mathrm{D}}\right) \times 100$, wherein $\mathrm{Q}_{\text {в }}$ was the amount of compounds in the receiver compartment $(\mu \mathrm{mol})$; $\mathrm{C}_{\mathrm{S}}$ was the donor concentration of test compounds $(\mu \mathrm{mol} / \mathrm{L})$; $\mathrm{V}_{\mathrm{D}}$ was the volume of the donor compartment $(\mathrm{L})$. The transport rate $\left(\mu \mathrm{mol} / \mathrm{h} / \mathrm{cm}^{2}\right)$ was calculated using the equation transport rate $=\mathrm{Q}_{\mathrm{B}} /(\mathrm{t} \times \mathrm{A})$, wherein $\mathrm{Q}_{\mathrm{B}}$ was the amount of compounds in the receiver compartment $(\mu \mathrm{mol})$; $\mathrm{t}$ was the incubating time $(\mathrm{h})$; $\mathrm{A}$ was the surface area of Caco-2 monolayer $\left(\mathrm{cm}^{2}\right)$.

The results presented in this study were the averages of at least three replicates and were presented as means $\pm \mathrm{SD}$. The data were analyzed by either $t$ test or nonparametric test after analysis of variance using SPSS 16.0. The level of significance was set at $p<0.05$.

\section{Conclusions}

In summary, the present results provided a basis for the establishment of the major contributors to the efficacy of the flowers of $T$. chinensis and supplied useful information to predict the oral bioavailability of these phenolic acids.

\section{Acknowledgments}

This work was supported by NSFC (No. 81073018 and No.81274044). We would like to express our gratitude to Xin-yuan Shi for her help in calculation of the lipo-hydro partition coefficients of the compounds. 


\section{Author Contributions}

Conceived and designed the experiments: Ru-Feng Wang and Xiu-Wen Wu. Performed the experiments: Xiu-Wen Wu and Li-Jia Liu. Analyzed the data: Can Zhao. Wrote the paper: Ru-Feng Wang and Xiu-Wen Wu. Helped edit the manuscript: Li-Na Guo.

\section{Conflicts of Interest}

The authors declare no conflict of interest.

\section{References}

1. Wang, R.F.; Yang, X.W.; Ma, C.M.; Liu, H.Y.; Shang, M.Y.; Zhang, Q.Y.; Cai, S.Q.; Park, J. Trollioside, a new compound from the flowers of Trollius chinensis. J. Asian Nat. Prod. Res. 2004, 6, 139-144.

2. Liu, Y.; Wang, R.F.; Yuan, M.; An, Y.N.; Wu, X.W. HPLC Assay of trollioside in flowers of Trollius chinensis. Chin. J. Exp. Tradit. Med. Formulae 2012, 18, 73-75.

3. Yuan, M.; Wang, R.F.; Wu, X.W.; An, Y.N.; Yang, X. Investigation on Flos Trollii: Constituents and bioactivities. Chin. J. Nat. Med. 2013, 11, 1-7.

4. Li, Y.L.; Ma, S.C.; Yang, Y.T.; Ye, S.M.; But, P.P. Antiviral activities of flavonoids and organic acid from Trollius chinensis Bunge. J. Ethnopharmacol. 2002, 79, 365-368.

5. Yuan, M. The Transformation Production of the Constituents in the Flowers of Trollius Chinensis by Human Intestinal Bacteria and Their Antibacterial Activity. Master thesis, Beijing University of Chinese Medicine, Beijing, China, 2013.

6. Li, Q.F.; Feng, S.Q.; Li, Y.L.; Cen, Y.Z.; Yang, Y.T.; Wang, L.Y. Study on the antibacterial and antiviral activity compositions of Trollius chinensis Bunge. J. Zhejiang Univ. (Sci. Ed.) 2004, 31, 412-415.

7. Wang, R.F. The Constituents and Bioactivities of Jinlianhua and Pangdahai. Doctoral Thesis, Peking University, Bejing, China, 2003.

8. Yang, X.W.; Yang, X.D.; Wang, Y.; Ma, L.; Zhang, Y.; Yang, X.G.; Wang, K. Establishment of Caco-2 cell monolayer model and standard operation procedure for assessing intestinal absorption of chemical components of traditional Chinese medicine. J. Chin. Integr. Med. 2007, 5, 634-641.

9. Artursson, P.; Karlsson, J. Correlation between oral drug absorption in humans and apparent drug permeability coefficients in human intestinal epithelial (Caco-2) cells. Biochem. Biophys. Res. Commun. 1991, 175, 880-885.

10. Yee, S.Y. In vitro permeability across Caco-2 cells (colonic) can predict in vivo (small intestinal) absorption in man-fact or myth. Pharm. Res. 1997, 14, 763-766.

11. Kerns, E.H.; Di, L. Drug-like Properties: Concepts, Structural Design and Methods; Science Press: Beijing, China, 2011; pp. 33-41.

Sample Availability: Samples of the compounds PA, GA and TS are available from the authors.

(C) 2014 by the authors; licensee MDPI, Basel, Switzerland. This article is an open access article distributed under the terms and conditions of the Creative Commons Attribution license (http://creativecommons.org/licenses/by/4.0/). 